\section{Diagnostic incisional biopsies in clinically indeterminate choroidal tumours}

S Seregard, C All-Ericsson, L Hjelmqvist,

L Berglin and A Kvanta
Abstract

Most intraocular tumours are reliably diagnosed by a careful clinical examination combined with one or more non-invasive diagnostic techniques. However, in a small percentage of tumours, typically small and clinically amelanotic, the features are insufficiently distinct for a confident clinical diagnosis and tissue is required for diagnosis. We used a $23-\mathrm{G}$ vitreous cutter to access the biopsy site in 43 patients with clinically indeterminate tumours. After retinotomy, an incisional choroidal biopsy yielded a specimen of $\sim 1 \mathrm{~mm}^{3}$. Obtained tissue was routinely processed for light microscopy including an immunohistochemical panel of monoclonal antibodies. Adequate tissue for diagnosis was provided in $41 / 43(95 \%)$ patients. The sensitivity and specificity to detect malignant disease were 0.97 and $\mathbf{1 . 0 0}$, respectively. The positive predictive value was 1.00. Complications included progression of pre-existing retinal detachment in $5 / 43(12 \%)$ patients and transient rise in intraocular pressure to $>40 \mathrm{~mm} \mathrm{Hg}$ in $6 / 43$ (14\%) patients; 4 of these 6 patients had a pre-existing retinal detachment. No patient with a pre-operatively attached retina had a retinal detachment. We conclude that an incisional transretinal choroidal biopsy yields abundant material and may adequately confirm or exclude malignancy in patients with clinically indeterminate tumours. The complication rate can be minimised when patients with pre-existing retinal detachment are excluded from biopsy. Eye (2013) 27, 115-118; doi:10.1038/eye.2012.219; published online 16 November 2012

Keywords: incision; biopsy; diagnosis; indeterminate; uveal melanoma; choroidal metastasis
Introduction

The diagnosis of primary cancer in nearly all sites of the body is routinely made by cytological or histopathological examination. Cancer of the central nervous system including the eye is a notable exception; in Sweden >95\% of cancer in all sites except tumours of the brain and eye has morphological confirmation. In contrast, less than half of primary intraocular cancer has morphological confirmation (typically after enucleation of the eye). ${ }^{1}$ This is because intraocular cancer (in the Western world largely comprising uveal melanoma and metastases to the choroid) is often reliably diagnosed by clinical examination and a range of non-invasive techniques and because of concern for ocular complications or extraocular seeding of tumour cells following tumour sampling. ${ }^{2-4}$ However, in a subset of patients harbouring a clinically amelanotic tumour without a history of metastatic extraocular cancer, a clinical diagnosis can be extremely challenging.

Intraocular biopsies are made for diagnostic, confirmatory (when the physician or the patient wishes to have a highly likely clinical diagnosis validated by cytological or histopathological examination), investigational or prognostic purposes. ${ }^{5}$ We have previously reported on a surgical technique used for clinically indeterminate intraocular lesions in adults to diagnose primary or secondary malignant disease. ${ }^{6}$ Although this technique is currently used only for a small subset of indeterminate lesions, numbers now allow for assessment of the diagnostic accuracy. Herein, we present the sensitivity and specificity to detect malignant disease using this incisional, transretinal, choroidal biopsy for clinically indeterminate tumours.

\section{Materials and methods}

A total of 46 patients had choroidal incisional biopsies for diagnostic purposes during a
Department of Vitreoretinal Diseases, St Eriks Eye Hospital and Karolinska Institutet, Stockholm Sweden

Correspondence: S Seregard, Department of Vitreoretinal Diseases, St Eriks Eye Hospital and Karolinska Institutet, Polhemsgatan 50, SE11282

Stockholm, Sweden

Tel: +46 86723000 ;

Fax: +46 96723375

E-mail: stefan.seregard@ sankterik.se

Received: 24 September 2012

Accepted in revised form: 1 October 2012

Published online:

16 November 2012

This work was presented at the 2012 Cambridge Ophthalmological Symposium and at the 2012 meeting of the Club Jules Gonin. 
10.5-year period. Two of these patients had the procedure undertaken because the patient insisted on histopathological confirmation before therapy was initiated. As both of these patients had characteristic lesions, which were felt to be reliably diagnosed clinically, they were classified as confirmatory and excluded. One more patient did not have a final diagnosis and was also excluded from the study.

A standard 23-G/20 G three-port pars plana vitrectomy was performed using the Alcon Accurus and Constellation vitrectomy systems (Alcon, Fort Worth, TX, USA). Endoillumination was provided by a $25-\mathrm{G}$ chandelier light to allow bimanual tumour tissue removal. An initial core vitrectomy was performed to gain access to the tumour and, if not present preoperatively, a posterior vitreous detachment was induced with the vitreous cutter followed by complete removal of the posterior hyaloid. The biopsy site was carefully chosen usually at the location of maximum tumour thickness in the posterior fundus and, if possible, peripheral to the temporal vascular arcades. When a serous detachment was present, a tumour site including attached retina was preferred. Also, tumour areas containing haemorrhage or fibrosis were avoided. To minimise bleeding, diathermy was applied to the retina overlying the biopsy site followed by a retinal incision of $\sim 1 \times 1 \mathrm{~mm}$ using a $20-\mathrm{G}$ diamond knife. The retinal tissue overlying the tumour was excised using a vitreous cutter. To minimise tumour bleeding, the intraocular pressure was temporarily raised after which the tumour was incised with a diamond knife. The tumour sample $\left(\sim 1 \mathrm{~mm}^{3}\right)$ was grasped with an intraocular end-gripping forceps and the sample base cut with the diamond knife. The specimen was then carefully removed through the sclerotomy during which infusion was stopped. After removal of the sample, the fluid infusion was re-administered and endolaser photocoagulation was applied around the biopsy site. A complete fluid-air exchange was made and the sclerotomies were sealed with absorbable sutures. Finally, intraocular air was substituted with $20 \%$ sulphur hexafluoride. In cases with prominent serous detachment, subretinal fluid was drained through the retinotomy and $5000 \mathrm{cs}$ silicone oil was used as intraocular tamponade.

Specimens measured were routinely fixed in $4 \%$ formaldehyde for $\sim 24 \mathrm{~h}$ and paraffin-embedded. Briefly, sections were then cut at $4 \mu \mathrm{m}$ and stained with a panel of monoclonal antibodies routinely including Melan A, (1:50; Dako, Glostrup, Denmark), HMB-45 (1: 150; Dako), S-100 protein (1:2000; Dako), CD 68 (1:100; Dako), and AE 1/3 (1:100 Bio-Genex, Fremont, CA, USA) adhering to a standard immunohistochemistry protocol. Further, immunostains were added as deemed appropriate in the individual case.

\section{Results}

The included 23 men and 20 women had a median age of 65 years (range: 31-91 years). All tumours were located in the choroid posterior to the equator. The median tumour thickness was $4 \mathrm{~mm}$ (range: $2-12 \mathrm{~mm}$ ). A preoperative retinal detachment was present in 15/43 (35\%) patients; 3 of these patients also had an associated vitreous haemorrhage. The median period of follow-up after biopsy was 27 months (range: 1-128 months). Two patients had repeat incisional biopsies performed, and two more patients had a previous failed biopsy using a $25-G$ vitrectomy system with biopsies provided using the vitreous cutter. Adequate tissue for diagnosis was obtained in $41 / 43(95 \%)$ patients. The sensitivity and specificity to detect malignant disease were 0.97 and 1.00 , respectively. The positive predictive value was 1.00. There were no false-positive biopsies, but one patient with a solitary choroidal metastasis had insufficient material for diagnosis. The patient was later diagnosed with primary adenocarcinoma of the lung and eventually died with disseminated disease. Post-mortem histopathological examination of the eye confirmed the presence of an adenocarcinoma metastatic to the choroid.

In total, 24/43 (56\%) patients were diagnosed with a uveal melanoma. Enucleation was performed in 15 of these patients and 9 patients had ruthenium brachytherapy. Histopathological examination of the enucleated eyes confirmed the biopsy diagnosis of uveal melanoma (Figure 1). The 7/43 (16\%) patients with metastatic disease had primary tumours of the breast, lung, prostate, and spinal cord (Figures 2 and 3). One more patient had a large choroidal plasmocytoma. Subsequent clinical work-up detected a testicular plasmocytoma. Notably, there was no bone marrow involvement indicative of myeloma.

Retinal detachment progressed in 5/15 (33\%) patients with a pre-operative retinal detachment. Four of these patients were managed by silicon oil tamponade. No patient with pre-operatively attached retina developed retinal detachment. Six patients had a transient rise in intraocular pressure to $>40 \mathrm{~mm} \mathrm{Hg}$; four of these patients had a pre-existing retinal detachment. The association of complications with a pre-existing retinal detachment was statistically significantly $(P=0.014$; $\chi^{2}$ test). Extraocular tumour spread at the sclerotomy site was not clinically evident in any patient during the period of follow-up. Similarly, eyes enucleated after biopsy failed to show any tumour cells at the sclerotomy sites by histopathological examination.

\section{Discussion}

The much improved non-invasive diagnosis of choroidal tumours now renders morphological confirmation 

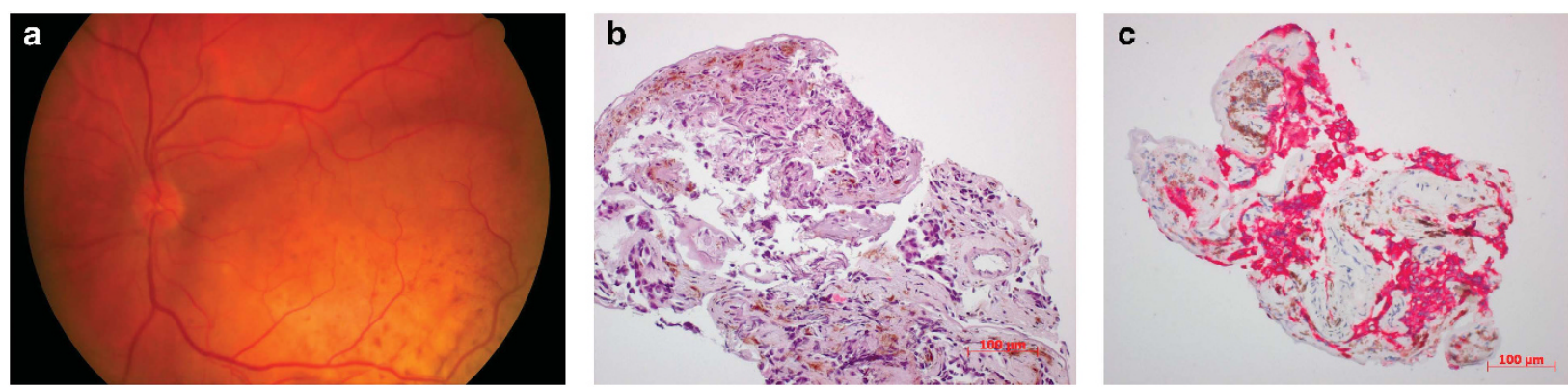

Figure 1 A 65-year-old man with a history of hyperthyroidism and thyroid eye disease experienced acute onset left periorbital oedema. Ophthalmological examination of the left eye disclosed a $14 \times 12 \times 5$-mm pale lesion in the posterior choroid (a). A routinely haematoxylin and eosin-stained section after biopsy showed abundant tumour cells, some fibrosis, and a few pigmented retinal pigment epithelial cells (b). Immunohistomical investigation revealed that the tumour cells were distinctly positive for an immunostain detecting a range of cytokeratins (AE 1/3). Note that the epithelial tumour cells are unevenly distributed, suggesting that a fine-needle aspiration biopsy may not necessarily have detected them (c). Additional clinical work-up showed a small carcinoma of the lung, and the patient later died of widespread disease.
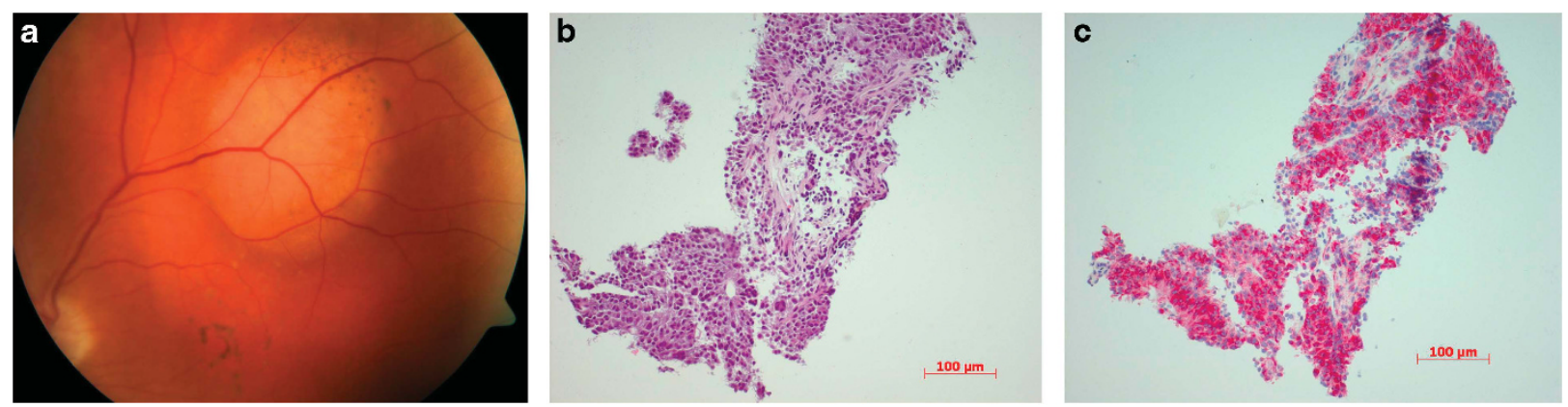

Figure 2 A 69-year-old woman with a left eye paracentral scotoma for $\sim 1$ year and a history of nephrectomy for renal adenocarcinoma 10 years earlier. Left eye fundus photograph shows a $4 \times 3 \times 2$-mm lesion with some mottling of the retinal pigment epithelium (a). Routine histopathology features solid growth of small tumour cells stained by haematoxylin and eosin (b). The tumour cells are uniformly and distinctly positive for melanoma-specific marker HMB-45 (c).
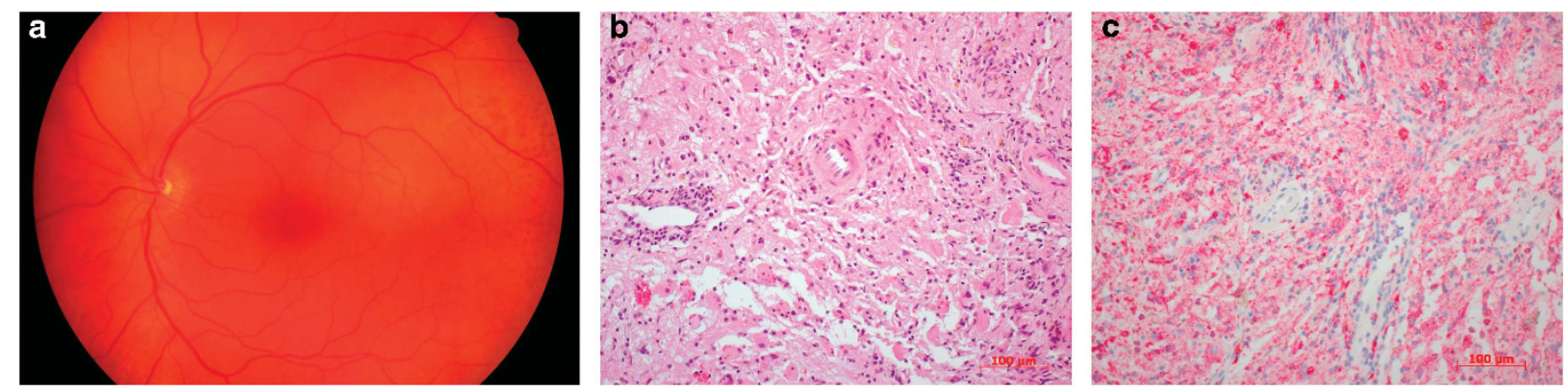

Figure 3 A 54-year-old woman experienced left eye blurred vision for 1 month. Fundus examination revealed a left eye, upper temporal, $5 \times 4 \times 2$-mm pale lesion adjacent to the fovea (a). Histopathological examination of a section stained with haematoxylin and eosin shows abundant macrophage-like cells and a few interspersed blood vessels and lymphocytes (b). Immunohistochemical staining with the CD68 marker confirmed the presence of numerous macrophages (c). A clinical work-up has so far been negative for any extraocular disease.

infrequent. For a number of reasons, there is a paucity of data on diagnostic accuracy in biopsies of indeterminate choroidal tumours. First, many biopsies reported as diagnostic are actually conformational as a clinical diagnosis highly likely to be correct was made before biopsy. In such series, the diagnostic sensitivity and specificity are, not surprisingly, likely to be high. Second, most series of diagnostic choroidal biopsies are based on fine-needle aspirates, but typically these are performed using a variety of transscleral, transretinal (transvitreal), or transcorneal routes to access the tumour. Third, only a few previous series are sufficiently large to include 
calculations of sensitivity, specificity, and the positive predictive value.

Recently, Faulkner-Jones et $a l^{7}$ reported a sensitivity and specificity of 0.96 and 0.83 , respectively, for detecting malignancy when using fine-needle aspiration biopsies for sampling. The positive predictive value was 0.96 . Most of these biopsies $(27 / 33 ; 82 \%)$ were in fact confirmatory, and a variety of routes were used to access the choroid. It appears that our data compare favourably in terms of diagnostic accuracy. Other techniques may yield larger samples than fine-needle aspiration biopsies and have been reported by Bechrakis et $a l^{8}$ using a $20-\mathrm{G}$ vitreous cutter and later by Sen et $a l^{9}$ using a $25-\mathrm{G}$ vitrectomy system. The basic difference between these techniques and ours is that they relied on a vitreous cutter to provide multiple, smaller, pieces of tumour tissue, but we used an incisional approach to secure a single (although sometimes actually multiple) tissue block(s) of some $1 \mathrm{~mm}^{3}$. Previous results from sampling by a vitreous cutter indicate a high yield in that only $1 / 23$ and $1 / 14$ cases were inadequate for diagnosis. 8,9 Similarly, our own results show that inadequate sampling only occurred in 2/43 cases. Importantly, we had no problem sampling thin tumours of $2 \mathrm{~mm}$ or less in thickness using the incisional approach. Notably, these are among the most difficult tumours to sample adequately using a vitreous cutter or fine-needle aspiration biopsy. ${ }^{4}$ Sensitivity, specificity, and positive predictive calculations were not provided in earlier series using the vitreous cutter approach. This makes comparison with our series difficult.

It is conceivable that a larger sample size may be associated with more complications. Indeed, when including all samples, complications appear to have been more frequent with our technique, but the number could be minimised when excluding patients with a preexisting retinal detachment. Moreover, any complications occurring in our series were manageable by standard vitreoretinal techniques. Intraocular or extraocular tumour spread is an infrequent event after fine-needle aspiration biopsies. Although, our sample size was significantly larger, none of the patients in our series had an extraocular tumour recurrence. In conclusion, our results suggest that incisional transretinal biopsies for indeterminate choroidal tumours are safe and accurate. Complications are infrequent when patients with a pre-existing retinal detachment are excluded.

\section{Conflict of interest}

The authors declare no conflict of interest.

\section{References}

1 Seregard S. To biopsy or not to biopsy? Acta Ophthalmol 2009; 87: 586-587

2 Midena E, Parrozzani R. Biopsies in uveal melanoma. In: Jager MJ, Desjardins L, Kivelä T, Damato BE (eds). Current Concepts in Uveal Melanoma. Karger: Basel, 2012, pp 81-95.

3 Augsburger JJ. Diagnostic biopsy of selected intraocular tumors. Am J Opthalmol 2005; 140: 1094-1095.

4 Eide N, Walaas L. Fine-needle aspiration biopsy and other biopsies in suspected intraocular malignant disease: a review. Acta Ophthalmol 2009; 83: 588-601.

5 Augsburger JJ, Correa ZM. Biopsy of iris tumors. Am J Ophthalmol 2011; 152: 720-722.

6 Kvanta A, Seregard S, Dafgard Kopp E, All-Ericsson C, Landau I, Berglin L. Choroidal biopsies for intraocular tumors of inderterminate origin. Am J Ophthalmol 2005; 140: 1002-1006.

7 Faulkner-Jones BE, Foster WJ, Harbour JW, Smith ME, Dávila RM. Fine needle aspiration biopsy with adjunct immunohistochemistry in intraocular tumor management. Acta Cytol 2005; 49: 297-307.

8 Bechrakis NE, Foerster MH, Bornfeld N. Biopsy in indeterminate intraocular tumors. Ophthalmology 2002; 109: 235-242.

9 Sen J, Groenewald C, Hiscott PS, Smith PA, Damato BE. Transretinal choroidal tumor biopsy with a 25-gauge vitrector. Ophthalmology 2006; 113: 1028-1031. 